\title{
COMPARISON OF LEICA BLK360 AND LEICA BLK2GO ON CHOSEN TEST OBJECTS
}

\author{
A. Dlesk ${ }^{1} *$, K. Vach ${ }^{2}$, J. Šedina ${ }^{1}$, K. Pavelka ${ }^{1}$ \\ ${ }^{1}$ Department of Geomatics, Faculty of Civil Engineering, Czech Technical University in Prague, 16629 Prague, Czech Republic - \\ (adam.dlesk, jaroslav.sedina, pavelka)@fsv.cvut.cz \\ ${ }^{2}$ EuroGV, spol. s r. o., 11000 Prague, Czech Republic - vach@eurogv.cz
}

Commission V, WG V/7

KEY WORDS: Leica BLK360, Leica BLK2GO, point cloud, laser scanning, BIM modelling.

\begin{abstract}
:
The contribution deals with the comparison of two laser scanners manufactured by Leica company. In BIM modelling, there is a need for fast and accurate gathering of spatial data, e.g. point clouds. Those data can be gathered by photogrammetry or laser scanning. Last years on the market, there occurred some light and easy-to-use alternatives to classic laser scanners. There were chosen two scanners that belong to the easy-to-use category. The first scanner is stationary Leica BLK360 and the second scanner is Leica BLK2GO which is a handheld scanner based on SLAM technology. Both laser scanners were tested on three different test objects. The first object is an administrative building, the second object is a historical administrative building and the third object is the vaults of the church. In all cases, only the indoor side of the objects was measured. The point clouds were compared to each other and the comparison was discussed. The parameters derived from the point clouds were also compared to the parameters read in the original documentation of the object. The comparison of the parameters may show, how those point clouds are usable for the final BIM modelling.
\end{abstract}

\section{MANUSCRIPT}

\subsection{Introduction}

Building information modelling (BIM) is a work process that is already implemented in many construction and facility management projects. Nowadays, BIM modelling is usually carried out during building design, planning, and construction. The model then contains a variety of important information. At the end of the construction processes, the rich BIM model can be handed over to the facility managers and the managers use the model for day-to-day processes of the building. Lots of buildings in the world have historical character and were constructed before the implementation of BIM. The need for the reconstruction or modernization of those buildings requires reverse BIM modelling. In those cases, it is necessary to provide some method of reverse engineering of the building. It is necessary to gather geometrical and descriptive information about the building. As a prevailing method of the reverse modelling is "scan to BIM" technology. The "scan to BIM" technology is a process of transferring the laser scan data into BIM models (Wang, 2019). The laser scanning process is usually carried out using modern laser scanners which have the ability to capture a lot of spatial data in a short time and create the point cloud. The laser scanning method is possible to substitute by photogrammetry where the final result is also a point cloud. According to the praxis, it is often convenient to use a combination of both methods. The point cloud is possible to import to the CAD software with BIM modelling support where it is possible to create a $3 \mathrm{D}$ model which can be enriched by the descriptive information.

The aim of this contribution is to present a comparison of different easy-to-use laser scanners produced by a single manufacturer on different case studies. One of the scanners is a relatively cheap compact stationary laser scanner Leica BLK360 which is according to the literature considered as suitable and reliable (Luhmann, 2019). In past years, there has been a development of scanners that are based on SLAM (Simultaneous Localization and Mapping) technology. Those scanners are handheld and it is possible to use them for continuous scanning during walking. Using those scanners, it is possible to capture a large amount of data in a very short time (compared to the stationary scanners). The SLAM scanner which is used for this contribution is Leica BLK2GO. The two scanners were used for scanning three different test objects. One of the objects is a family house, the second is a historical administrative building and the third one is a church vaults. The objects were scanned using the mentioned laser scanners and the point clouds were analyzed and compared to each other. This contribution tries to bring an answer if the faster laser scanner Leica BLK2GO is comparable to Leica BLK360 in terms of accuracy.

\section{INSTRUMENTS}

For point cloud generation, two instruments manufactured by a Leica company were chosen. The first chosen scanner was compact stationary scanner Leica BLK360, the second chosen scanner was SLAM scanner BLK2GO.

\subsection{Leica BLK360}

Leica BLK360 is a compact laser scanner. The scanner is relatively small and very light (around $1 \mathrm{~kg}$ ). The compactness is the best advantage of the scanner. The scanner together with the tripod and remote controller (tablet or smartphone) is possible to place into a single small case. The compactness does not bring too high compromises in the scanner resolution,

\footnotetext{
* Corresponding author
} 
accuracy, and efficiency. The range of the scanner is from $0.6 \mathrm{~m}$ to $60 \mathrm{~m}$ with a ranging accuracy of $4 \mathrm{~mm}$ at $10 \mathrm{~m}$ from the scanner and $7 \mathrm{~mm}$ at $20 \mathrm{~m}$ from the scanner. There are three different selectable resolution settings $-5 \mathrm{~mm}, 10 \mathrm{~mm}$, and 20 $\mathrm{mm}$ at $10 \mathrm{~m}$. The scanner is equipped with a 15-megapixel camera and gives the opportunity to create a colored point cloud and $360^{\circ}$ panorama image. The scanner is relatively fast. At the lowest resolution $(20 \mathrm{~mm}$ at $10 \mathrm{~m})$ without capturing a digital image, the measuring time is just 40 seconds. This gives a potential for a very fast scan to BIM processes. The scanner is possible to control with an application Leica Cyclone FIELD 360. With the application, it is possible to register the setups of the measurement directly in the field. So, the point cloud is preprocessed before returning to the office. Over that, the scanner is also equipped with a thermal camera which can create a thermal infrared panoramic image in $360^{\circ} \times 70^{\circ}$.

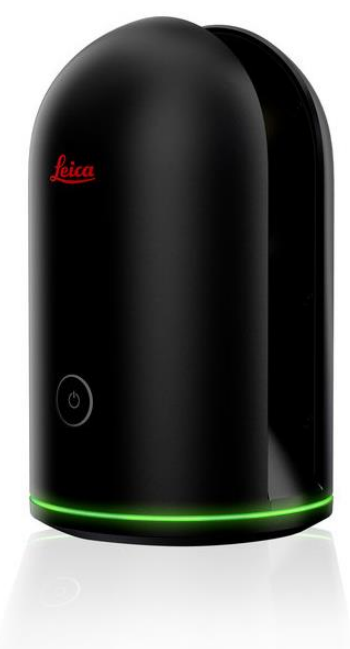

Figure 1. Laser scanner Leica BLK360 (Leica Geosystems, 2022).

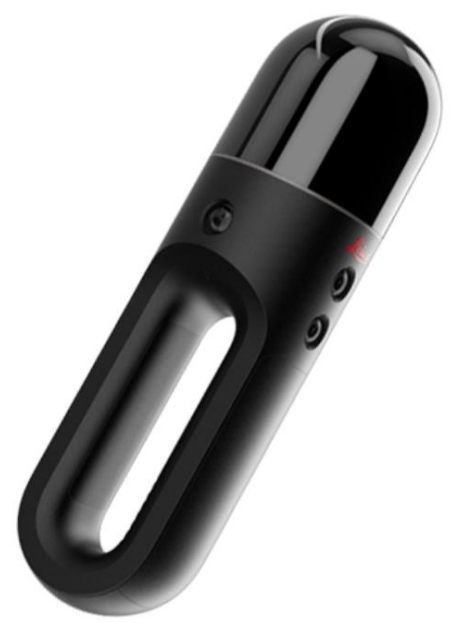

Figure 2. Laser scanner Leica BLK2GO (Gefos a.s., 2022).

\subsection{Leica BLK2GO}

Leica BLK2GO is a handheld laser scanner that is based on SLAM technology. Using the device, it is possible to scan during walking in real-time. Every time during scanning, the device measure points of the point cloud and capture the images (panoramic). The scanner is equipped with three panoramic cameras which identify corresponding points and supported by the Inertial Measurement Unit (IMU) can calculate the position of the scanner in the space in real-time. Simply, the huge advantage of the scanner is time efficiency. The scanner has the ability to capture 420000 points per second. The range of the scanner is from $0.5 \mathrm{~m}$ up to $25 \mathrm{~m}$. The noise range is $\pm 3 \mathrm{~mm}$. The laser scanner BLK2GO is possible to control using the mobile application "BLK2GO live". During the laser scanner walks, the operator checks the trajectory of the walk on the device screen. The scanner is possible to transport in the light and small case.

\section{LASER SCANNING OF TEST OBJECTS}

Three test objects were chosen for the comparison of the instruments. First, the small administrative building was chosen (Test Object 1), the second object is a historical administrative building (Test Object 2), and the third object were vaults of the historical church (Test Object 3). Only an interior was scanned for all the objects. All the objects are in daily use and in the interior, there were objects like furniture which made the measurement more complicated and brought unwanted points for further modelling. All objects were scanned using the Leica BLK360 and Leica BLK2GO laser scanners. During the measuring, the panoramic images were taken as well.

The resolution of the Leica BLK360 measurement was set at the lowest $2 \mathrm{~cm}$ at $10 \mathrm{~m}$. This resolution was chosen to increase the speed of laser scanning. For interior mapping, the distances are around $5 \mathrm{~m}$ and lower so for $2 \mathrm{D}$ and $3 \mathrm{D}$ modelling seemed the chosen resolution as sufficient. Measuring of one setup with Leica BLK360 was 1 minute and 40 seconds. The setups were co-registered together in the application Leica Cyclone FIELD 360 during the measuring.

Leica BLK2GO was used using the mobile application BLK2GO Live. The application has controlled the trajectory of the walks. Because of the battery and the data size of the resulting point clouds of the walks, the longest walk was taken around 6 minutes (approx. $4 \mathrm{~GB}$ ). The walks were created with the significant overlap between each other. The walks were coregistered together in the office using the desktop application Leica Cyclone REGISTER 360. It is important to note that it was not easy to find overlapping parts and register the walks together because the 6 minutes walks were huge and there were many times several rooms even on a few building storeys.

The different measuring time costs in the field using different laser scanners are presented in Tab 1 . The presented time costs are not just the time costs when the instruments were scanning but with all necessities which the job in the field requires. From the table, it is obvious that Leica BLK2GO has a huge advantage in time cost. The following analysis and comparison may show if Leica BLK2GO can compete with Leica BLK360 even in terms of the accuracy of the result.

\begin{tabular}{|c|c|c|c|c|}
\hline & \multicolumn{2}{|c|}{ BLK360 } & \multicolumn{2}{c|}{ BLK2GO } \\
\hline & setups & time & walks & time \\
\hline Test & 44 & $4 \mathrm{~h} 18 \mathrm{~m}$ & 4 & $0 \mathrm{~h} 20 \mathrm{~m}$ \\
$\begin{array}{c}\text { Obj. 1 } \\
\text { Test }\end{array}$ & 92 & $7 \mathrm{~h} 14 \mathrm{~m}$ & 10 & $1 \mathrm{~h} 50 \mathrm{~m}$ \\
$\begin{array}{c}\text { Obj. 2 } \\
\text { Test } \\
\text { Obj. 3 }\end{array}$ & 56 & $6 \mathrm{~h} 19 \mathrm{~m}$ & 7 & $37 \mathrm{~min}$ \\
\hline
\end{tabular}

Table 1. Time costs of using different instruments. 


\section{CLOUD-TO-CLOUD COMPARISON ON ADMINISTRATIVE BUILDINGS}

For test object 1 and test object 2 were used Leica BLK360 and Leica BLK2GO instruments. For the analysis of this paper, the point clouds generated from the two scanners were compared together. Before the comparison, the point clouds were filtered in Geomagic software. To filter the outliers, the Select Outliers function with parameter 0.85 was used. For the noise reduction, the function Reduce Noise with Prismatic Shapes filter was used (Smooth level $=1$ and number of iteration $=2$ ). The point clouds created by Leica BLK2GO contained more noise and outliers. The filtering function reduced those point clouds on average by $19 \%$. On the other hand, the point clouds created by Leica BLK360 were reduced on average by $12 \%$.

For the comparison, point cloud from Leica BLK360 was used as the reference one and point cloud from Leica BLK2GO was always compared one. For a better cloud-to-cloud comparison between point cloud from Leica BLK360 and Leica BLK2GO, the test object $n .2$ was divided into several parts. Because of the compactness and small dimension of the test object num. 1, the object was not divided. So, the compared point clouds were:

1. Test object num. 1

2. $2^{\text {nd }}$ basement

3. $1^{\text {st }}$ basement

4. Ground floor

5. $1^{\text {st }}$ floor

6. Stairs

7. Doors

8. Windows

9. Ceilings

The cloud-to-cloud comparison was carried out in Cloud Compare software. In each cloud-to-cloud comparison, the standard error was calculated. The calculated standard errors are presented in Table 2.

\begin{tabular}{|l|c|}
\hline Cloud-to-cloud & $\sigma[\mathrm{mm}]$ \\
\hline Test object n. 1 & 14 \\
$2^{\text {nd }}$ basement & 12 \\
$1^{\text {st }}$ basement & 12 \\
Ground floor & 12 \\
$1^{\text {st }}$ floor & 13 \\
Stairs & 11 \\
Doors & 17 \\
Windows & 21 \\
Ceilings & 18 \\
\hline
\end{tabular}

Table 2. Calculated standard errors of cloud-to-cloud comparison between point cloud from Leica BLK360 and Leica BLK2GO.

According to the table, the standard error of cloud-to-cloud comparison on larger point clouds (test object and storeys) was between $12 \mathrm{~mm}$ and $14 \mathrm{~mm}$. In point clouds which represent doors, windows, and ceilings, the standard error was larger, up to $21 \mathrm{~mm}$. It is important to note that the accuracy of Leica BLK2GO is $4 \mathrm{~mm}$ and the accuracy of registration of the point cloud together is estimated as $6 \mathrm{~mm}$.

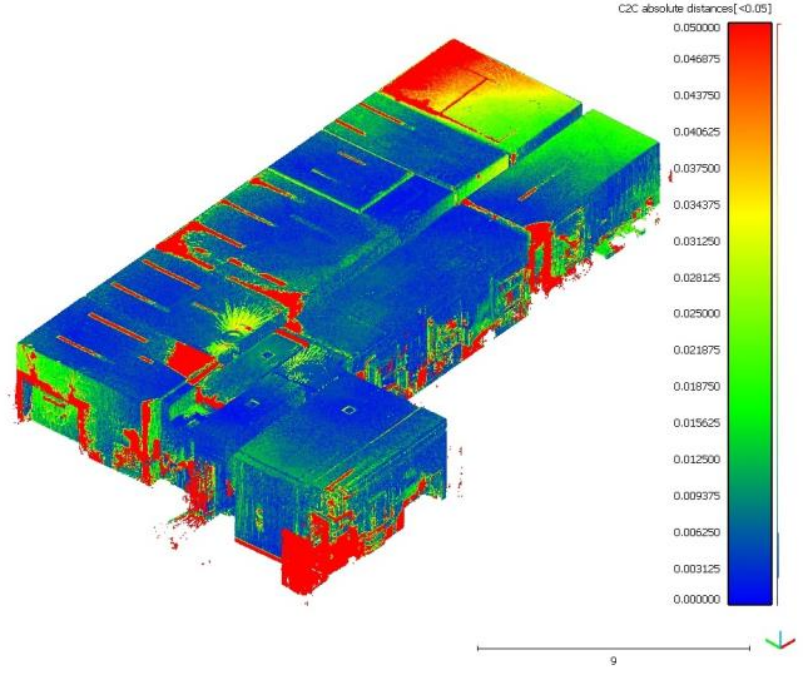

Figure 3. Cloud-to-cloud comparison of the $2^{\text {nd }}$ basement of the test object n. 2 .

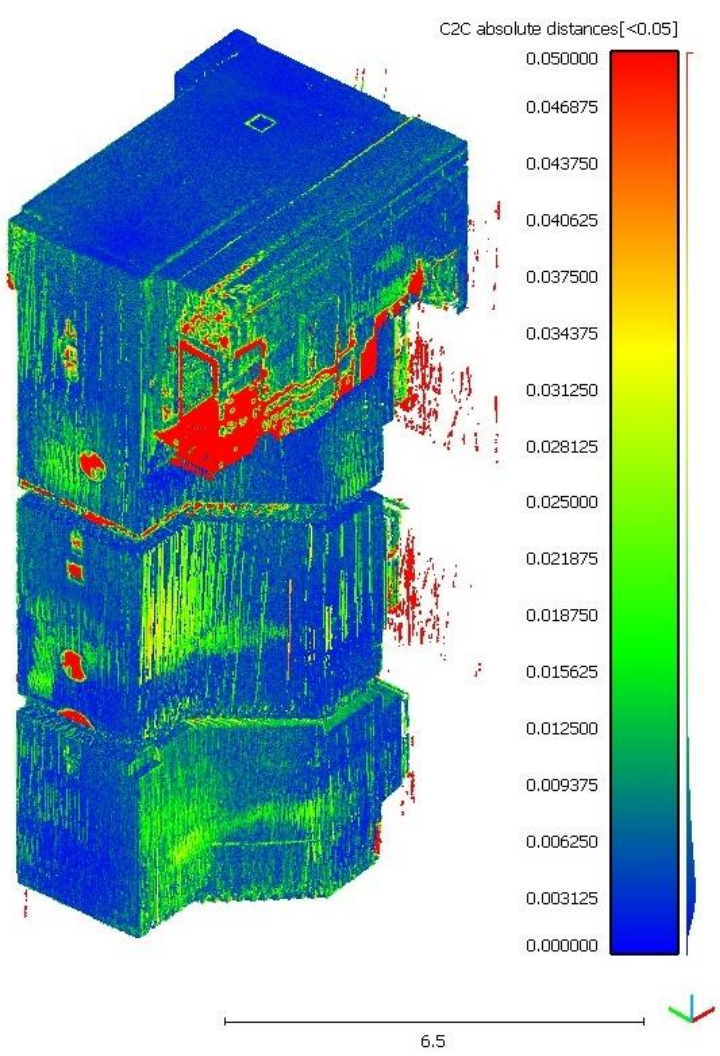

Figure 4. Cloud-to-cloud comparison on point cloud of stairs at the test object n. 2 . 


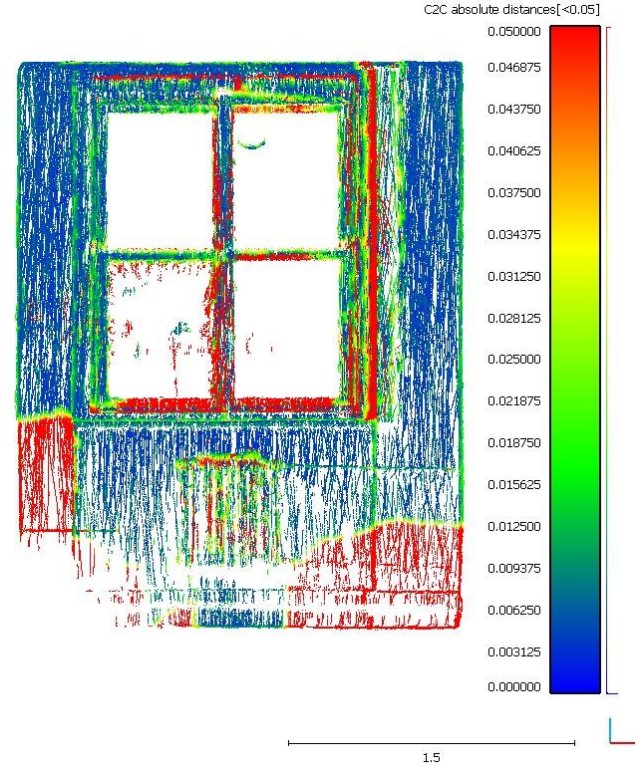

Figure 5. Cloud-to-cloud comparison on point cloud of a window at the test object n. 2 .

\subsection{Comparison with the original documentation}

The laser scanning data is possible to use for BIM modelling. To present the potential of the scanners for BIM modelling, a comparison was carried out. At the test object n. 2, from the point clouds from Leica BLK360 and Leica BLK2GO were derived parameters of the construction objects and those parameters were compared to the original documentation (DOC). Among the observed parameters were areas of the rooms, lengths, and widths of rooms, resulting in a thickness of the walls, heights, widths of windows and doors, and heights of the sills. This comparison may determine if the laser scanning data from different instruments are useable for 2D and 3D BIM modelling. The comparison may show if there is expected a difference in "scan to BIM" process when is used using Leica BLK360 or Leica BLK2GO. Overall, it was quite easy to derive the parameters of the rooms and doors. But on the test object n. 2 , there are complicated window objects. It was possible to determine the window width but for the certain derivation of window height and sill height the scanning resolution was not enough in this case.

\begin{tabular}{|l|c|c|c|}
\hline \multicolumn{1}{|c|}{$\left[\mathrm{m}^{2}\right]$} & $\begin{array}{c}\text { DOC. }- \\
\text { BLK360 }\end{array}$ & $\begin{array}{c}\text { DOC. }- \\
\text { BLK2GO }\end{array}$ & $\begin{array}{c}\text { BLK360- } \\
\text { BLK2GO }\end{array}$ \\
\hline Average & 0.24 & 0.25 & 0.01 \\
Std. deviation & 0.15 & 0.08 & 0.12 \\
Minimum & 0.01 & 0.10 & -0.32 \\
Maximum & 0.73 & 0.43 & 0.24 \\
Range & 0.33 & 0.72 & 0.56 \\
\hline
\end{tabular}

Table 3. Comparison of areas (number of observations $=30$ ).

According to Table 1., the area derived from the point cloud by Leica BLK2GO and Leica BLK360 is systematically lower than the area read from the original documentation. The fact that the comparison between the areas from both scanners has an average of around zero, points that there is a certain inaccuracy in the original documentation. In this case, there is a higher range in the parameters derived from the Leica BLK2GO point cloud.

\begin{tabular}{|l|c|c|c|}
\hline \multicolumn{1}{|c|}{$[\mathrm{m}]$} & DOC. - & DOC. - & BLK360- \\
& BLK360 & BLK2GO & BLK2GO \\
\hline Average & -0.001 & -0.026 & -0.025 \\
Std. deviation & 0.023 & 0.021 & 0.007 \\
Minimum & -0.036 & -0.061 & -0.041 \\
Maximum & 0.047 & 0.021 & -0.005 \\
Range & 0.083 & 0.082 & 0.036 \\
\hline
\end{tabular}

Table 4. Comparison of lengths (number of observation $=30$ ).

In the case of lengths (Table 2.), there is a certain systematic error at the point cloud by Leica BLK2GO. The average error is around $2.5 \mathrm{~cm}$. The average of observation from point cloud by Leica BLK360 is much lower $-0.001 \mathrm{~m}$. The standard deviation and the range of the set of observations are the same for both instruments.

\begin{tabular}{|l|c|c|c|}
\hline \multicolumn{1}{|c|}{$[\mathrm{m}]$} & $\begin{array}{c}\text { DOC. }- \\
\text { BLK360 }\end{array}$ & $\begin{array}{c}\text { DOC. }- \\
\text { BLK2GO }\end{array}$ & $\begin{array}{c}\text { BLK360- } \\
\text { BLK2GO }\end{array}$ \\
\hline Average & -0.003 & 0.025 & 0.028 \\
Std. deviation & 0.017 & 0.017 & 0.010 \\
Minimum & -0.042 & -0.010 & -0.013 \\
Maximum & 0.028 & 0.066 & 0.043 \\
Range & 0.070 & 0.076 & 0.056 \\
\hline
\end{tabular}

Table 5. Comparison of wall thickness (number of observations $=30$ ).

The wall thickness was another examined parameter for the following modelling. Even here the parameter derived from Leica BLK2GO point cloud shows a certain systematic error (average is $2.5 \mathrm{~cm}$ ). Even in this case, the average of observation from the point cloud by Leica BLK360 is much lower.

Another way how to compare the original documentation to the point clouds is graphical check. Laser scanners by Leica BLK360 and BLK2GO are suitable for fast and efficient checks of the quality of the original documentation. By this check, it is simply possible to find obvious and more detailed errors. In many cases, it is uncertain under which conditions and with what method with different accuracy was the documentation carried out. Simply, the sections of the point clouds are overlayed over the original documentation. According to the example detail on Fig. 6, the graphical check proved that the original documentation is done well.

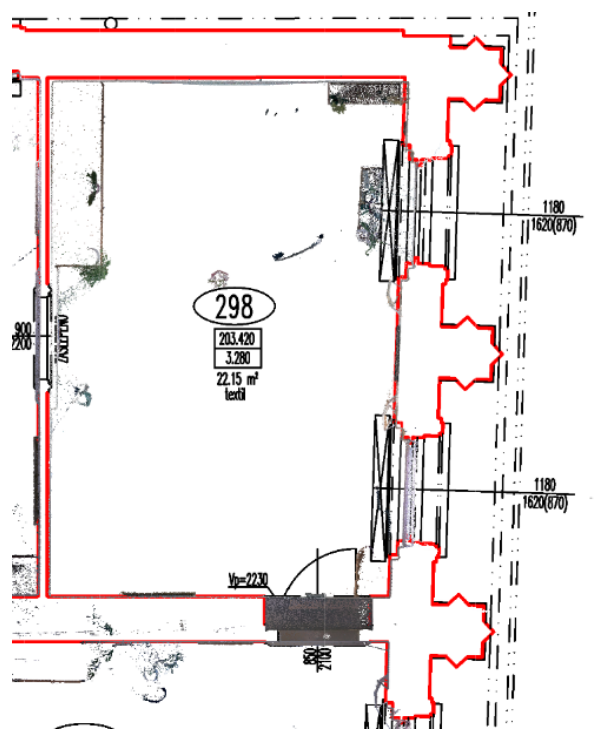

Figure 6. Detail of comparison of point cloud carried out Leica BLK360 and original documentation. 


\section{CLOUD-TO-CLOUD COMPARISON ON CHURCH VAULTS}

The laser scanners Leica BLK360 and Leica BLK2GO were used also for scanning the church vaults. The church vaults were chosen because the vaults are in height around $11 \mathrm{~m}$ from the floor and the laser scanners can be tested for longer distances. The distances on previous objects were around $5 \mathrm{~m}$ and usually less than $5 \mathrm{~m}$.

Both point clouds were pre-processed in Cloud Compare software. At first, the statistical outlier removal function (SOR filter in Cloud Compare) was applied. Then, the subsampling of the point cloud has been carried out. The point cloud from Leica BLK360 was supposed to be used as the reference one, so the point cloud was subsampled for a better resolution of 1 point per ca. $2 \mathrm{~mm}^{2}$. The point cloud from Leica BLK2GO was supposed to be compared to the point cloud from Leica BLK360, so the point cloud was subsampled to a resolution of 1 point per ca. $4 \mathrm{~mm}^{2}$. The point clouds were co-registered. Then, the point clouds were compared together using cloud-tocloud comparison. The mean distance of the cloud-to-cloud comparison was $0.015 \mathrm{~m}$ with a standard deviation of $0.042 \mathrm{~m}$. According to Figure 7., the largest errors were mainly on the edges of the vault and on the vault decorations. Most of the points $(90 \%)$ were under $2 \mathrm{~cm}$. This shows a high similarity between the point cloud by Leica BLK360 and Leica BLK2GO. $96 \%$ of the points were under $5 \mathrm{~cm}$. The rest of the points can be considered as outliers which should be manually or automatically filtered from the point cloud. The testing showed that even though the measuring with Leica BLK2GO was very fast (37 min) compared to the measuring with Leica BLK360 (more than 6 hours), the accuracy is comparable.

\section{CONCLUSIONS}

The implementation of BIM processes at already built constructions requires the development of reverse engineering techniques. The BIM geometry modelling requires spatially oriented data, usually point clouds. From the point cloud, it is possible to create a BIM model in advanced CAD software. The point cloud is usually obtained by photogrammetry or laser scanning. Due to the development of SLAM technology, there has been introduced new handheld laser scanner. Handheld laser scanners have a major advantage. The measuring with those scanners is much more faster than using conventional stationary laser scanners. In this paper, the question if the reduced time cost is compromised by lower accuracy has been asked.

For the purposes of this paper, there has been compared the results from the two instruments, both manufactured by Leica company. One of them is the stationary laser scanner Leica BLK360 and the second one is the handheld laser scanner Leica BLK2GO. The laser scanners have been compared on three test objects - administrative building, historic administrative building and vaults of the church. The measuring distances in both administrative buildings were short (around $5 \mathrm{~m}$ ). The church vaults have been chosen to test the accuracy of the measurement on larger distance around $10 \mathrm{~m}$. The point clouds which were acquired by both laser scanners were compared to each other and then were compared to the original documentation of the objects. All the comparisons and analysis showed that the accuracy of the results from Leica BLK2GO laser scanner was comparable to the accuracy of the results from the Leica BLK360 laser scanner (on the selected test objects). When the speed of measuring by Leica BLK2GO is taken into an account, it can be pointed out that LeicaBLK2GO can be for similar objects convenient and brings a big benefit for the scan to BIM processes. Also, the laser scanning data is possible to use for the quick accuracy check of the original documentation or the $3 \mathrm{D}$ modelling. Checking the correctness of $2 \mathrm{D}$ or $3 \mathrm{D}$ models should be an integral part of BIM processes.
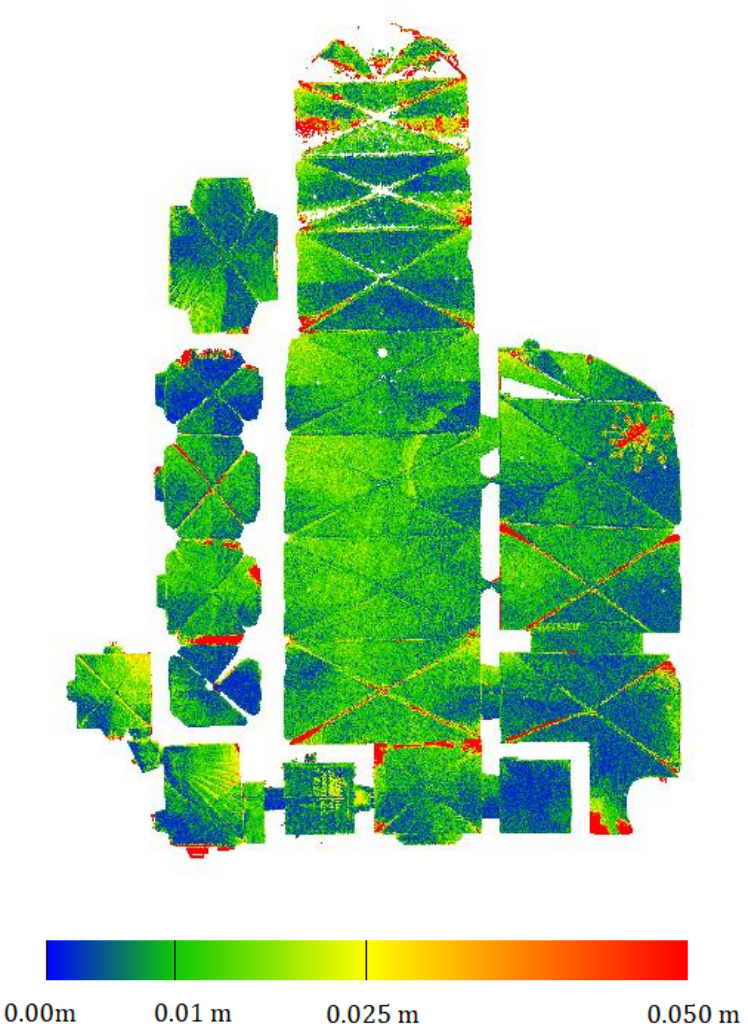

Figure 7. Cloud-to-cloud comparison of the church between a point cloud from Leica BLK360 and a point cloud from Leica BLK2GO.

\section{ACKNOWLEDGEMENTS}

This project was supported by the European structural and investment funds - Operational Programme Prague - Growth Pole of the Czech Republic n. CZ.07.1.02/0.0/0.0/16_025/0000605. Also, we would like to thank GEFOS a.s. for borrowing us laser scanner Leica BLK2GO.

\section{REFERENCES}

Gefos a.s. 3D skener BLK2GO. 2022. https://www.gefosleica.cz/novinky/detail-clanku-278-3d-skener-blk2go

Grussenmeyer, P. et al., 2008. Comparison Methods of Terrestrial Laser Scanning, Photogrammetry and Tacheometry Data for Recording of Cultural Heritage Buildings. The International Archives of Photogrammetry, Remote Sensing and Spatial Information Sciences, 37(B5), p. 213-218, Beijing.

Luhmann, T., Chizhova M., Gorkovchuk D., Hastedt H., Chachava N., Lekveishvili N., 2019: Combination of terrestrial laserscanning, UAV and close-range photogrammetry for 3D reconstruction of complex churches in Georgia. Int. Arch. Photogramm. Remote Sens. Spatial Inf. Sci., XLII-2/W11, 753761. doi.org/10.5194/isprs-archives-XLII-2-W11-753-2019. 
Luhmann, T.; Robson, S.; Kyle, S.; Harley, I., 2007. Close range photogrammetry. Wiley.

Leica Geosystems. Leica BLK360. 2022. https://shop.leicageosystems.com/buy/blk360/blk360.

Wang, Q., Guo, J., Kim, M. K. 2019. An application oriented scan-to-BIM framework. Remote sensing, 11(3), 365. 